\title{
Chloroquine retinopathy: Is there a safe daily dose?
}

\author{
J. S. MARKS
}

From the Rheumatism Research Centre, Royal Infirmary, Manchester M139WL

SUMMARY Published case reports of chloroquine retinopathy rarely include details of daily dosage, but 30 reports where this information was available included 78 patients who developed impaired visual acuity and 13 had received daily doses of $250 \mathrm{mg}$ or less. Eleven cases of ocular toxicity attributed to chloroquine were reported to the Committee on Safety of Medicines but only 2 developed impaired vision on $250 \mathrm{mg}$ daily. A postal questionnaire among 41 rheumatology and 33 ophthalmology centres showed 2 patients who developed impaired vision as a result of treatment with chloroquine in a daily dose not exceeding $250 \mathrm{mg}$. Serious visual impairment related to chloroquine rarely occurs if the daily dose does not exceed $250 \mathrm{mg}$.

The beneficial effect of chloroquine in the treatment of rheumatoid arthritis was established by long-term controlled trials, ${ }^{12}$ but reports of retinal toxicity resulted in the drug falling into disfavour. ${ }^{3}$ The incidence of chloroquine retinopathy has varied from less than $1 \%{ }^{4}$ to $16 \%$ in different series depending on the techniques used to establish the diagnosis.

Early studies suggested that the risk of chloroquine retinopathy is related to the total dose and total duration of treatment, ${ }^{6-9}$ and this cumulative effect may be explained by its slow excretion ${ }^{10}$ and preferential accumulation in the pigmented layers of the eye. ${ }^{11}$ In animals the risk of chloroquine retinotoxicity is related to the daily dose, ${ }^{12}$ and this correlates with the nonlinear rapid increase in chloroquine binding to melanin at increasing concentrations of the drug. ${ }^{13}$ Mackenzie and Scherbel ${ }^{14}$ suggested that the rate of administration governs the incidence of retinopathy, and there is a threshold level for the daily dose below which visual impairment does not occur irrespective of the cumulative drug consumption or the duration of treatmeni. In a recent retrospective study ${ }^{15}$ on ophthalmic complications in 222 patients on longterm treatment with chloroquine a reduction in visual acuity was found in only 1 patient who had received $500-750 \mathrm{mg}$ daily, and this suggested that the risk of serious visual impairment is negligible provided the daily dose does not exceed $250 \mathrm{mg}$. In the present study an attempt has been made to confirm or refute this observation, because it would have important implications on the necessity for regular ophthalmic supervision.

Accepted for publication 24 February 1981

Correspondence to Dr J. S. Marks.
This question cannot be resolved by a prospective study, since it would be unethical to continue treatment, in spite of possible abnormalities in retinal screening tests, until visual acuity became impaired. The alternative is to ascertain retrospectively those patients who developed visual impairment while on chloroquine with particular reference to the daily dose they had been receiving.

\section{Materials and methods}

All case reports of chloroquine retinopathy published in English were reviewed and the relationship between daily dose and ocular toxicity assessed from those reports in which the daily dose was stated and visual acuity was impaired. ${ }^{68915-41}$

Adverse reactions to chloroquine reported to the Committee on Safety of Medicines (CSM) were reviewed with particular reference to the daily dose that had been used in those developing ocular complications.

A postal questionnaire was sent to 45 British rheumatology and 76 ophthalmology centres requesting information on the daily dose of chloroquine that had been used in any of their patients developing visual impairment while on treatment. The centres contacted were distributed throughout England and Scotland to take account of possible regional differences in susceptibility and criteria of ocular toxicity.

\section{Results}

There were 78 individual case reports among 30 publications on chloroquine retinopathy in which it 
Table 1 Review of published reports of chloroquine retinopathy with impaired vision

\begin{tabular}{|c|c|c|c|c|c|c|c|c|c|}
\hline \multirow[t]{2}{*}{ Authors } & \multirow[t]{2}{*}{ Diagnosis } & \multirow[t]{2}{*}{ Age } & \multirow[t]{2}{*}{ Sex } & \multirow{2}{*}{$\begin{array}{l}\text { Daily dose } \\
(m g)\end{array}$} & \multirow{2}{*}{$\begin{array}{l}\text { Total } \\
\text { duration } \\
\text { (years) }\end{array}$} & \multicolumn{2}{|c|}{ Visual Acuity } & \multirow[t]{2}{*}{ Field loss } & \multirow[t]{2}{*}{ Macular changes } \\
\hline & & & & & & $R$ & $L$ & & \\
\hline $\begin{array}{l}\text { Cambiaggi } 16 \\
\text { Hobbs et al. } .^{17}\end{array}$ & SLE & 37 & $\mathbf{F}$ & 500 & 2 & $20 / 40$ & $20 / 30$ & Constricted to $10^{\circ}$ & Pigmented \\
\hline $\begin{array}{l}\text { No. } 1 \\
\text { No. } 2 \\
\text { No. } 3\end{array}$ & $\begin{array}{l}\text { DLE } \\
\text { RA } \\
\text { Arthritis }\end{array}$ & $\begin{array}{l}68 \\
60 \\
66\end{array}$ & $\begin{array}{l}\mathbf{F} \\
\mathbf{F} \\
\mathbf{F}\end{array}$ & $\begin{array}{l}100-600 \\
200-300^{*} \\
400\end{array}$ & $\begin{array}{l}3 \frac{1}{2} \\
3 \\
3\end{array}$ & $\begin{array}{l}6 / 9 \\
6 / 9 \\
6 / 18\end{array}$ & $\begin{array}{l}6 / 6 \\
6 / 9 \\
6 / 12\end{array}$ & $\begin{array}{l}\text { Paracentral } \\
\text { Paracentral } \\
\text { Peripheral }\end{array}$ & $\begin{array}{l}\text { Pigmented } \\
\text { Atrophic } \\
\text { Oedema }\end{array}$ \\
\hline Wells ${ }^{18}$ & DLE & 26 & $\mathbf{F}$ & $200^{*}$ & 2 & $6 / 18$ & $6 / 9$ & Central & Diffuse pigmentation \\
\hline Hobbs et al. 19 & DLE & 43 & $\mathbf{F}$ & $400-600$ & 3 & $6 / 9$ & $6 / 12$ & Peripheral & Oedema \\
\hline $\begin{array}{l}\text { Richards and Zeller20 } \\
\text { Ellsworth and Wilson }{ }^{21}\end{array}$ & RA & 42 & $\mathbf{F}$ & 500 & $2 \frac{1}{2}$ & $20 / 40$ & $20 / 40$ & Peripheral & Oedema \\
\hline $\begin{array}{l}\text { No. } 1 \\
\text { No. } 2 \\
\text { Price22 }\end{array}$ & $\begin{array}{l}\text { SLE } \\
\text { RA }\end{array}$ & $\begin{array}{l}22 \\
34\end{array}$ & $\begin{array}{l}\mathbf{F} \\
\mathbf{F}\end{array}$ & $\begin{array}{l}1000 \\
200-300\end{array}$ & $\begin{array}{l}3 t \\
2\end{array}$ & $\begin{array}{l}20 / 30 \\
20 / 30\end{array}$ & $\begin{array}{l}20 / 30 \\
20 / 30\end{array}$ & $\begin{array}{l}\text { Paracentral } \\
\text { Paracentral }\end{array}$ & $\begin{array}{l}\text { Pigmentation } \\
\text { Pigmentation }\end{array}$ \\
\hline $\begin{array}{r}\text { Price } 22 \\
\text { No. } 1 \\
\text { No. } 2 \\
\text { No. } 3 \\
\text { No. } 4 \\
\text { No. } 5 \\
\text { No. } 6\end{array}$ & $\begin{array}{l}\text { RA } \\
\text { SLE } \\
\text { SLE } \\
\text { DLE } \\
\text { Arthritis } \\
\text { Arthritis }\end{array}$ & $\begin{array}{l}45 \\
40 \\
34 \\
66 \\
75 \\
31\end{array}$ & $\begin{array}{l}\mathbf{F} \\
\mathbf{F} \\
\mathbf{F} \\
\mathbf{F} \\
\mathbf{F} \\
\mathbf{F}\end{array}$ & $\begin{array}{l}750 \\
600 \\
750 \\
400 \\
400 \\
250-500\end{array}$ & $\begin{array}{l}1 \\
1 \frac{1}{2} \\
5 \\
5 \\
\text { 'Years' } \\
\text { 'Years' }\end{array}$ & $\begin{array}{l}6 / 18 \\
6 / 9 \\
6 / 9 \\
6 / 18 \\
6 / 60 \\
6 / 18\end{array}$ & $\begin{array}{l}6 / 12 \\
6 / 9 \\
6 / 36 \\
6 / 18 \\
6 / 12 \\
6 / 24\end{array}$ & $\begin{array}{l}\text { Paracentral } \\
\text { Peripheral } \\
- \\
\text { Peripheral } \\
\text { Paracentral }\end{array}$ & $\begin{array}{l}\text { Pigmented } \\
\text { Pigmented } \\
\text { Degeneration } \\
\text { Optic atrophy } \\
\text { Optic atrophy } \\
\text { Pigmented }\end{array}$ \\
\hline $\begin{array}{l}\text { George \& Mitchell } \\
1962(23)\end{array}$ & SLE & 37 & $\mathbf{F}$ & $200-600$ & 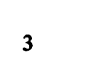 & $6 / 6$ & $6 / 9$ & Contracted & Pigmented \\
\hline Ormrod 24 & & & & & & & & & \\
\hline $\begin{array}{l}\text { No. } 1 \\
\text { No. } 2\end{array}$ & $\begin{array}{l}\text { SLE } \\
\text { Skin disease }\end{array}$ & $\begin{array}{l}28 \\
53\end{array}$ & $\begin{array}{l}\mathbf{M} \\
\mathbf{M}\end{array}$ & $\begin{array}{l}300-500 \\
200-400\end{array}$ & $\begin{array}{l}4 \\
7\end{array}$ & $\begin{array}{l}6 / 9 \\
6 / 12\end{array}$ & $\begin{array}{l}6 / 9 \\
6 / 12\end{array}$ & $\begin{array}{l}\text { Ring } \\
\text { Ring }\end{array}$ & $\begin{array}{l}\text { Pigmented } \\
\text { Pigmented }\end{array}$ \\
\hline Penner and Somers 25 & DLE & 42 & $\mathbf{F}$ & $500-1000$ & 3 & $20 / 30$ & $20 / 30$ & Paracentral & Pigmented \\
\hline Mayer 26 & $\mathbf{R A}$ & 30 & $\mathbf{F}$ & 250 & 31 & $20 / 30$ & $20 / 30$ & Ring & Pigmented \\
\hline $\begin{array}{l}\text { Reed and Campbel127 } \\
\text { Smith28 }\end{array}$ & RA & 55 & $\mathbf{F}$ & 250 & 1 & $20 / 120$ & $20 / 120$ & Central & Normal \\
\hline $\begin{array}{l}\text { No.1 } \\
\text { No.2 } \\
\text { No. } 3\end{array}$ & $\begin{array}{l}\text { Scleroderma } \\
\text { RA } \\
\text { RA }\end{array}$ & $\begin{array}{l}52 \\
45 \\
48\end{array}$ & $\begin{array}{l}\mathbf{M} \\
\mathbf{F} \\
\mathbf{F}\end{array}$ & $\begin{array}{l}400^{*} \\
250 \\
500\end{array}$ & $\begin{array}{l}2^{\frac{1}{2}} \\
2 \frac{1}{2}\end{array}$ & $\begin{array}{l}20 / 200 \\
20 / 40 \\
20 / 400\end{array}$ & $\begin{array}{l}20 / 50 \\
20 / 40 \\
20 / 400\end{array}$ & $\begin{array}{l}\text { Central } \\
\text { Pericentral } \\
\quad-\end{array}$ & $\begin{array}{l}\text { Bull's eyes } \\
\text { Bull's eyes } \\
\text { Degeneration }\end{array}$ \\
\hline Jansson 29 & & & & & & & & & \\
\hline $\begin{array}{l}\text { No. } 1 \\
\text { No. } 2\end{array}$ & $\begin{array}{l}\text { DLE } \\
\text { RA }\end{array}$ & $\begin{array}{l}60 \\
53\end{array}$ & $\begin{array}{l}\mathbf{M} \\
\mathbf{F}\end{array}$ & $\begin{array}{l}200-400 \\
250-500\end{array}$ & $\begin{array}{l}5 \\
2 \frac{1}{2}\end{array}$ & $\begin{array}{l}6 / 9 \\
6 / 60\end{array}$ & $\begin{array}{l}6 / 9 \\
6 / 60\end{array}$ & $\begin{array}{l}\text { Pericentral } \\
\text { Pericentral }\end{array}$ & $\begin{array}{l}\text { Pigmented } \\
\text { Pigmented }\end{array}$ \\
\hline $\begin{array}{l}\text { Algvere et al. }{ }^{30} \\
\text { No. } 1 \\
\text { No. } 2\end{array}$ & $\begin{array}{l}\text { RA } \\
\text { SLE }\end{array}$ & $\begin{array}{l}55 \\
40\end{array}$ & $\begin{array}{l}\mathbf{M} \\
\mathbf{M}\end{array}$ & $\begin{array}{l}500 \\
400-600\end{array}$ & $\begin{array}{l}3 \frac{1}{2} \\
3\end{array}$ & $\begin{array}{l}6 / 9 \\
6 / 60\end{array}$ & $\begin{array}{l}6 / 6 \\
6 / 18\end{array}$ & $\begin{array}{l}\text { Pericentral } \\
\text { Pericentral }\end{array}$ & $\begin{array}{l}\text { Bull's eye } \\
\text { Pigmented }\end{array}$ \\
\hline $\begin{array}{l}\text { Okun et al. }{ }^{31} \\
\text { No. } 1 \\
\text { No. } 2 \\
\text { No. } 3 \\
\text { No. } 4 \\
\text { No. } 5 \\
\text { No. } 6 \\
\text { No. } 7 \\
\text { No. } 8\end{array}$ & $\begin{array}{l}\text { DLE } \\
\text { DLE } \\
\text { DLE } \\
\text { DLE } \\
\text { Asthma } \\
\text { DLE } \\
\text { DLE } \\
\text { DLE }\end{array}$ & $\begin{array}{l}50 \\
39 \\
49 \\
40 \\
53 \\
54 \\
52 \\
39\end{array}$ & $\begin{array}{l}\mathbf{F} \\
\mathbf{F} \\
\mathbf{M} \\
\mathbf{F} \\
\mathbf{M} \\
\mathbf{F} \\
\mathbf{F} \\
\mathbf{F}\end{array}$ & $\begin{array}{l}250-500 \\
250-500 \\
500-750^{*} \\
250-500^{*} \\
250-375^{*} \\
500 \\
250-750^{*} \\
500\end{array}$ & $\begin{array}{l}8 \frac{1}{2} \\
5 \frac{1}{1} \\
5 \frac{1}{2} \\
1 \\
4 \\
6 \frac{1}{2} \\
3 \frac{1}{2} \\
3\end{array}$ & $\begin{array}{l}20 / 30 \\
20 / 200 \\
20 / 30 \\
20 / 30 \\
20 / 40 \\
7 / 400 \\
20 / 50 \\
15 / 200\end{array}$ & $\begin{array}{l}20 / 25 \\
20 / 50 \\
20 / 20 \\
20 / 25 \\
20 / 50 \\
11 / 400 \\
20 / 500 \\
4 / 200\end{array}$ & $\begin{array}{l}\text { Pericentral } \\
\text { Pericentral } \\
\text { Pericentral } \\
\text { Ring } \\
\text { Pericentral } \\
\text { Central } \\
\text { Peripheral } \\
\text { Small islands of } \\
\quad \text { vision }\end{array}$ & $\begin{array}{l}\text { Granular } \\
\text { Bull's eye } \\
\text { Bull's eye } \\
\text { Pigmented } \\
\text { Pigmented } \\
\text { Pigmented } \\
\text { Diffuse pigment } \\
\text { Diffuse pigment }\end{array}$ \\
\hline $\begin{array}{l}\text { Elliot and Mills } 32 \\
\text { No. } 1 \\
\text { No. } 2 \\
\text { Crews }^{33}\end{array}$ & $\begin{array}{l}\text { RA } \\
\text { RA }\end{array}$ & $\begin{array}{l}61 \\
47\end{array}$ & $\mathbf{F}$ & $\begin{array}{l}250-500^{*} \\
500\end{array}$ & $\begin{array}{l}6 \\
2 \frac{1}{2}\end{array}$ & $20 / 25$ & $\begin{array}{r}10 / 200 \\
5 / 400\end{array}$ & $\begin{array}{l}\text { Central } \\
\text { Pericentral }\end{array}$ & $\begin{array}{l}\text { Degeneration } \\
\text { Pigmentation }\end{array}$ \\
\hline $\begin{array}{l}\text { No. } 1 \\
\text { No. } 2\end{array}$ & $\begin{array}{l}\text { RA } \\
\text { SLE }\end{array}$ & $\begin{array}{l}68 \\
72\end{array}$ & $\mathbf{F}$ & $\begin{array}{l}400-800 \\
500\end{array}$ & $1 \frac{3}{2}$ & $\begin{array}{l}6 / 6 \\
6 / 9\end{array}$ & $\begin{array}{l}6 / 36 \\
6 / 9\end{array}$ & $\begin{array}{l}\text { Central } \\
\text { Peripheral }\end{array}$ & $\begin{array}{l}\text { Pigmented } \\
\text { Diffuse pigment and } \\
\text { optic atrophy }\end{array}$ \\
\hline $\begin{array}{l}\text { Henkind et al. }{ }^{34} \\
\text { No. } 1 \\
\text { No. } 2 \\
\text { No. } 3 \\
\text { Lewis }{ }^{35} \\
\text { Lloyd Hiltz } 36\end{array}$ & $\begin{array}{l}\text { SLE } \\
\text { SLE } \\
\text { DLE } \\
\text { RA }\end{array}$ & $\begin{array}{l}36 \\
40 \\
27 \\
64\end{array}$ & $\begin{array}{l}\mathbf{F} \\
\mathbf{F} \\
\mathbf{M} \\
\mathbf{F}\end{array}$ & $\begin{array}{l}500 \\
500 \\
500 \\
250-500\end{array}$ & $\begin{array}{l}6 \\
7 \\
3 \frac{1}{2} \\
7\end{array}$ & $\begin{array}{l}20 / 40 \\
20 / 50 \\
20 / 20 \\
20 / 40\end{array}$ & $\begin{array}{l}20 / 40 \\
20 / 20 \\
20 / 35 \\
20 / 40\end{array}$ & $\begin{array}{l}\text { Central } \\
\text { Nil } \\
\text { Central } \\
\text { Peripheral }\end{array}$ & $\begin{array}{l}\text { Granular } \\
\text { Pigmented } \\
\text { Pigmented } \\
\text { Atrophic discs }\end{array}$ \\
\hline No. 1 & SLE & 36 & $\mathbf{F}$ & 450 & 6 & $6 / 18$ & $\mathbf{C} / \mathbf{F}$ & $\begin{array}{l}\text { Bitemporal } \\
\text { hemianopia }\end{array}$ & Diffuse pigment \\
\hline $\begin{array}{l}\text { No. } 2 \\
\text { No. } 3\end{array}$ & $\begin{array}{l}\text { RA } \\
\text { RA }\end{array}$ & $\begin{array}{l}34 \\
58\end{array}$ & $\begin{array}{l}\mathbf{F} \\
\mathbf{F}\end{array}$ & $\begin{array}{l}250-500 \\
250-750\end{array}$ & $\begin{array}{l}4 \\
6\end{array}$ & $\begin{array}{l}6 / 18 \\
5 / 12\end{array}$ & $\begin{array}{l}6 / 18 \\
5 / 12\end{array}$ & $\begin{array}{l}\text { Paracentral } \\
\text { Ring }\end{array}$ & $\begin{array}{l}\text { Bull's eyes } \\
\text { Bull's eyes }\end{array}$ \\
\hline $\begin{array}{l}\text { Kearns and } \\
\text { Hollenhorst }^{37}\end{array}$ & & & & & & & & & \\
\hline $\begin{array}{r}\text { No. } 1 \\
\text { No. } 2 \\
\text { No. } 3 \\
\text { Burns } 38\end{array}$ & $\begin{array}{l}\text { RA } \\
\text { SLE } \\
\text { SLE }\end{array}$ & $\begin{array}{l}48 \\
56 \\
54\end{array}$ & $\begin{array}{l}\mathbf{F} \\
\mathbf{F} \\
\mathbf{F}\end{array}$ & $\begin{array}{l}500 \\
500 \\
500\end{array}$ & $\begin{array}{r}5 \\
6 \\
-\end{array}$ & $\begin{array}{l}20 / 30 \\
20 / 40 \\
20 / 30\end{array}$ & $\begin{array}{c}20 / 30 \\
20 / 40 \\
8 / 200\end{array}$ & $\begin{array}{l}\text { Pericentral } \\
\text { Ring } \\
\text { Ring }\end{array}$ & $\begin{array}{l}\text { Pigmented } \\
\text { Pigmented } \\
\text { Bull's eye }\end{array}$ \\
\hline $\begin{array}{l}\text { No. } 1 \\
\text { No. } 2\end{array}$ & $\begin{array}{l}\text { Epilepsy } \\
\text { DLE }\end{array}$ & $\begin{array}{l}14 \\
51\end{array}$ & $\mathbf{F}$ & $\begin{array}{l}250-750 \\
750\end{array}$ & $\frac{2 t}{2}$ & $\begin{array}{l}20 / 30 \\
20 / 60\end{array}$ & $\begin{array}{l}20 / 60 \\
20 / 20\end{array}$ & $\begin{array}{l}\text { Pericentral } \\
\text { Pericentral }\end{array}$ & $\begin{array}{l}\text { Bull's eye } \\
\text { Bull's eye }\end{array}$ \\
\hline
\end{tabular}


Table 1 -continued

\begin{tabular}{|c|c|c|c|c|c|c|c|c|c|}
\hline \multirow[t]{2}{*}{ Authors } & \multirow[t]{2}{*}{ Diagnosis } & \multirow[t]{2}{*}{ Age } & \multirow[t]{2}{*}{ Sex } & \multirow{2}{*}{$\begin{array}{l}\text { Daily dose } \\
(m g)\end{array}$} & \multirow{2}{*}{$\begin{array}{l}\text { Total } \\
\text { duration } \\
\text { (years) }\end{array}$} & \multicolumn{2}{|c|}{ Visual acuity } & \multirow[t]{2}{*}{ Field loss } & \multirow[t]{2}{*}{ Macular changes } \\
\hline & & & & & & $\boldsymbol{R}$ & $L$ & & \\
\hline \multicolumn{10}{|l|}{ Crews 39} \\
\hline No. 1 & RA & 55 & $\mathbf{F}$ & 500 & 3 & $6 / 18$ & $6 / 18$ & Annular & Diffuse pigment \\
\hline No. 2 & RA & 66 & $\mathbf{F}$ & 400 & 4d & $6 / 9$ & $6 / 9$ & Nil & Pre-retinopathy \\
\hline No. 3 & RA & 80 & $\mathbf{F}$ & 400 & 11 & $6 / 9$ & $6 / 9$ & Nil & Pre-retinopathy \\
\hline \multicolumn{10}{|l|}{ Carlberg 40} \\
\hline No. 1 & DLE & 48 & $\mathbf{M}$ & 600 & 3 & $6 / 18$ & $6 / 18$ & Pericentral & Bull's eye \\
\hline & $\mathbf{R A}$ & 50 & $\mathbf{F}$ & 250 & 5 & $6 / 12$ & $6 / 12$ & Pericentral & Bull's eye \\
\hline \multicolumn{7}{|l|}{ Nylander6 } & & & \\
\hline $\begin{array}{l}\text { No. } 1 \\
\text { No. } 2\end{array}$ & SLE & 46 & $\mathbf{F}$ & $250-500$ & 6 & $\begin{array}{l}0 / 12 \\
5 / 9\end{array}$ & $\begin{array}{l}5 / 9 \\
6 / 6\end{array}$ & Central & Oedema \\
\hline No. 3 & RA & 53 & $\mathbf{F}$ & 250 & 9 & $6 / 9$ & $\begin{array}{l}6 / 6 \\
6 / 18\end{array}$ & Central & Pigmented \\
\hline No. 4 & DLE & 63 & $\mathbf{M}$ & $250^{*}$ & $10 \frac{1}{2}$ & $6 / 9$ & $\begin{array}{l}6 / 18 \\
5 / 9\end{array}$ & Central & Oedema \\
\hline No. 5 & DLE & 61 & $\mathbf{M}$ & $200 *$ & 7 & $6 / 9$ & $3 / 9$ & Central & Degeneration \\
\hline No. 6 & SLE & 41 & $\mathbf{F}$ & 375 & $8 \frac{1}{2}$ & $6 / 9$ & $0 / 9$ & Tube & Pigment \\
\hline No. 7 & $\mathbf{R A}$ & 43 & $\mathbf{F}$ & 250 & $4^{\frac{1}{2}}$ & $6 / 9$ & $6 / 9$ & Peripheral & Atrophic \\
\hline No. 8 & SLE & 55 & $\mathbf{F}$ & 250 & $\begin{array}{l}4 \\
5\end{array}$ & $5 / 12$ & $5 / 12$ & Central & Bull's eye \\
\hline No. 9 & SLE & 30 & $\mathbf{F}$ & $250-500$ & 5 & $6 / 6$ & $5 / 12$ & Paracentral & Pigmented \\
\hline No. 10 & $\mathbf{R A}$ & 51 & $\mathbf{F}$ & $250-500$ & $5 \frac{1}{2}$ & $6 / 9$ & $6 / 9$ & Central & Pigmented \\
\hline No. 11 & RA & 44 & $\mathbf{F}$ & & 4 & $6 / 9$ & $6 / 6$ & Ring & Pigmented \\
\hline \multirow{2}{*}{\multicolumn{10}{|c|}{ Vorpio and Karjalainen 18}} \\
\hline No. 1 & SLE & 36 & $\mathbf{F}$ & 200 & 8 & & & & \\
\hline No. 2 & DLE & 50 & $\mathbf{F}$ & 250 & 5 & $5 / 9$ & $6 / 12$ & Pericentral & Granular \\
\hline \multicolumn{10}{|l|}{ Carr et al. 9} \\
\hline No. 1 & SLE & 41 & $\mathbf{F}$ & 500 & 8 & $20 / 20$ & $20 / 40$ & Pericentral & Pigmented \\
\hline No. 2 & SLE & 40 & $\mathbf{F}$ & 500 & 6 & $20 / 40$ & $20 / 40$ & Central & Bull's eyes \\
\hline \multicolumn{10}{|l|}{ Brinkley et al.41 } \\
\hline No. 1 & SLE & 47 & $\mathbf{F}$ & 420 & 3 & $6 / 9$ & $6 / 9$ & Constricted & Bull's eyes \\
\hline No. 2 & SLE & 63 & $\mathbf{F}$ & 405 & 4 & $6 / 9$ & $6 / 9$ & Constricted & Bull's eyes \\
\hline No. 3 & SLE & 59 & $\mathbf{F}$ & $500^{*}$ & 2 & $6 / 60$ & $6 / 30$ & Pericentral & Mottling \\
\hline No. 4 & SLE & 54 & $\mathbf{F}$ & $500^{*}$ & $1 \frac{1}{2}$ & $6 / 9$ & $6 / 9$ & Constricted & Mottling \\
\hline No. 5 & SLE & 62 & $\mathbf{F}$ & $500^{*}$ & $6 t$ & $6 / 7 \cdot 5$ & $6 / 9$ & Constricted & Normal \\
\hline Marks and Power ${ }^{15}$ & RA & 50 & $\mathbf{F}$ & $500-750$ & $4 \frac{1}{2}$ & $6 / 18$ & $1 / 60$ & Paracentral & Bull's eyes \\
\hline
\end{tabular}

*Other antimalarial therapy

was possible to examine the relationship between the daily dose and visual impairment (Table 1). Chloroquine retinopathy associated with visual impairment was reported in only 13 cases where the daily dose did not exceed $250 \mathrm{mg}$, and in 3 of these the patients had also received other antimalarials. The remaining case reports all referred to patients who had received more than $250 \mathrm{mg}$ daily: 47 received up to $500 \mathrm{mg}$ daily, 15 up to $750 \mathrm{mg}$ daily, and 3 more than $750 \mathrm{mg}$ daily.

There were 11 reports of chloroquine retinopathy to the CSM (Table 2). Five of these (cases 1, 2, 6, 8, 9) were stated to have visual impairment, but 2 (cases $8,9)$ developed eye symptoms after only a few days of treatment, and these were unlikely to be the result of a retinopathy. One patient (case 6) had been taking chloroquine intermittently for 2 years for malaria chemoprophylaxis and developed sudden deterioration in vision, but $i_{l}$ is unclear whether this was the result of a retinopathy. There were 2 patients (cases 1, 2) who developed an unquestionable chloroquine retinopathy associated with visual impairment, and both had received daily doses of $250 \mathrm{mg}$ or less.

A postal questionnaire was sent to 45 rheumatology centres and replies were received from 41 . Twenty-three centres had no cases of ocular toxicity, but 11 of these commented that chloro-

Table 2 Chloroquine retinopathy reports to Committee on Safety of Medicines

\begin{tabular}{|c|c|c|c|c|c|c|}
\hline Case & Diagnosis & Age & Sex & $\begin{array}{l}\text { Daily dose } \\
\text { (mg) }\end{array}$ & $\begin{array}{l}\text { Total duration } \\
\text { (years) }\end{array}$ & Ophthalmic findings \\
\hline $\begin{array}{r}1 \\
2 \\
3 \\
4 \\
5 \\
6 \\
7 \\
8 \\
9 \\
10 \\
11\end{array}$ & $\begin{array}{l}- \\
- \\
\overline{-} \\
\text { Malaria } \\
\text { Malaria } \\
\text { RA } \\
\overline{\text { RA }} \\
- \\
\text { - }\end{array}$ & $\begin{array}{l}53 \\
40 \\
45 \\
61 \\
50 \\
45 \\
43 \\
63 \\
64 \\
55\end{array}$ & $\begin{array}{l}\mathbf{F} \\
\mathbf{F} \\
\mathbf{F} \\
\mathbf{F} \\
\mathbf{M} \\
\mathbf{M} \\
\mathbf{F} \\
\mathbf{F} \\
\mathbf{M} \\
\mathbf{M}\end{array}$ & $\begin{array}{l}200 \\
250 \\
250 \\
400 \\
\text { 'Several' } \\
500 \text { Weekly } \\
- \\
500-750 \\
750 \\
250 \\
1000\end{array}$ & $\begin{array}{l}8 \\
5 \\
4 \\
2 \frac{1}{2} \\
10 \\
2 \\
9 \\
4 \text { days } \\
2 \text { days } \\
1 t \\
6 \text { weeks }\end{array}$ & $\begin{array}{l}\text { 6/36 6/24 Retinal degeneration } \\
\text { Retinopathy. Partial blindness } \\
\text { Maculat retinopathy } \\
\text { Retinopathy } \\
\text { 'Typical' retinopathy } \\
\text { Sudden deterioration in vision } \\
6 / 6 \text { 6/6 Macular degeneration } \\
\text { Acute eye symptoms } \\
\text { Blurred vision } \\
\text { Granular macula. Later tobacco amblyopia } \\
\text { Nonprogressive retinopathy }\end{array}$ \\
\hline
\end{tabular}


Table 3 Chloroquine retinopathy: Cases from rheumatology questionnaire

\begin{tabular}{|c|c|c|c|c|c|c|c|c|c|c|}
\hline \multirow[t]{2}{*}{ Case } & \multirow[t]{2}{*}{ Diagnosis } & \multirow[t]{2}{*}{ Age } & \multirow[t]{2}{*}{ Sex } & \multirow{2}{*}{$\begin{array}{l}\text { Daily } \\
\text { dose } \\
(m g)\end{array}$} & \multirow{2}{*}{$\begin{array}{l}\text { Total } \\
\text { duration } \\
\text { (years) }\end{array}$} & \multicolumn{2}{|c|}{ Visual Acuity } & \multirow[t]{2}{*}{ Field loss } & \multicolumn{2}{|c|}{ Macular changes } \\
\hline & & & & & & $\boldsymbol{R}$ & $L$ & & $\boldsymbol{R}$ & $L$ \\
\hline 1 & - & - & - & 500 & 4 & $6 / 18$ & $6 / 18$ & - & ++ & + \\
\hline 2 & - & 34 & $\mathbf{F}$ & '12 Tablets' & 4 & $6 / 60$ & $6 / 60$ & Central & + & + \\
\hline 3 & RA & 57 & $\mathbf{M}$ & 250 & $1 \frac{1}{2}$ & $6 / 24$ & $6 / 6$ & - & $++t$ & + \\
\hline 4 & RA & 53 & $\mathbf{F}$ & $100-600$ & $10^{2}$ & $6 / 12$ & $6 / 9$ & Ring & + & + \\
\hline 5 & RA & 72 & $\mathbf{F}$ & 250 & 2 weeks & - & - & - & ++ & ++ \\
\hline 6 & RA & 59 & $\mathbf{F}$ & 250 & $\frac{1}{2}$ & $6 / 9$ & $\mathbf{C} / \mathbf{F}$ & L central & + & ++ \\
\hline 7 & RA & 51 & $\mathbf{M}$ & 250 & $4 \frac{1}{2}$ & $6 / 12$ & $\mathbf{C} / \mathbf{F}$ & Paracentral & ++ & ++ \\
\hline 8 & SLE & 33 & $\mathbf{F}$ & 500 & 5 & $6 / 12$ & $6 / 60$ & & +++ & +++ \\
\hline 9 & - & 38 & $\mathbf{F}$ & 400 & 5 & $6 / 24$ & $6 / 12$ & Paracentral & +++ & +++ \\
\hline
\end{tabular}

+ Macular mottling. + + Bull's eye macula. ++ + Bull's eye and diffuse pigmentation.

Table 4 Chloroquine retinopathy: cases from ophthamology questionnaire

\begin{tabular}{|c|c|c|c|c|c|c|c|c|c|c|}
\hline \multirow[t]{2}{*}{ Case } & \multirow[t]{2}{*}{ Diagnosis } & \multirow[t]{2}{*}{ Age } & \multirow[t]{2}{*}{$\operatorname{sex}$} & \multirow{2}{*}{$\begin{array}{l}\text { Daily } \\
\text { dose } \\
(m g)\end{array}$} & \multirow{2}{*}{$\begin{array}{l}\text { Total } \\
\text { duration } \\
\text { (years) }\end{array}$} & \multicolumn{2}{|c|}{ Visual Acuity } & \multirow[t]{2}{*}{ Field loss } & \multicolumn{2}{|c|}{ Macular changes } \\
\hline & & & & & & $\boldsymbol{R}$ & $L$ & & $\boldsymbol{R}$ & $L$ \\
\hline
\end{tabular}

++ Bull's eye macula.

quine was rarely used. Eighteen centres had seen patients with ocular toxicity, and these included 17 cases of retinopathy without visual impairment and 13 cases of retinopathy with impaired vision. Adequate information on the daily dose was available in only 9 of these 13 cases (Table 3). Four patients had been receiving a daily dose of $250 \mathrm{mg}$, but in 2 (cases 3 and 6) the diagnosis of chloroquine retinopathy was doubtful because the ocular abnormalities were unilateral. One patient (case 5) developed bull's eye maculae after only 2 weeks of treatment and the retinal changes were probably coincidental to chloroquine therapy. Only 1 patient (case 7) appears to have developed a genuine chloroquine retinopathy on a dose of $250 \mathrm{mg}$ daily, but this patient had received chloroquine phosphate for 4 years until 1967, and in 1973 when seen by an optician was reported to have $6 / 6$ vision in both eyes. The chloroquine was resumed the following year, and routine ophthalmic assessment after 6 months showed 6/9 vision in both eyes. Although the chloroquine was there upon discontinued, his vision deteriorated over the next 4 years to $6 / 12$ and counting fingers.

A postal questionnaire was sent to 76 ophthalmology centres, and replies were received from 33. Twenty-six centres had no cases of ocular toxicity. Seven centres reported a total of 4 cases of retinopathy without visual impairment and 8 cases with impaired vision. Adequate information on the daily dose was available in only 3 of these 8 cases (Table
4). One patient (case 2) had taken chloroquine phosphate $250 \mathrm{mg}$ daily for 7 years for nocturnal cramps and developed typical features of chloroquine retinopathy associated with loss of vision. Another patient (case 3) who became blind was stated to have received chloroquine phosphate 100 mg twice daily, but tablets of chloroquine containing $100 \mathrm{mg}$ in chloroquine phosphate have never been available in the United Kingdom, and she was probably receiving $250 \mathrm{mg}$ twice daily.

\section{Discussion}

The major hazard associated with the use of chloroquine is retinopathy, which may be irreversible 172224 or may progress after treatment is discontinued. ${ }^{21} 31$ Many rheumatologists are reluctant to use antimalarials, and the British National Formulary 1976-8 condemns their use and states that chloroquine 'is now regarded as obsolete.'

The retinopathy may be reversible if diagnosed in the early stages, ${ }^{334243}$ but there is disagreement about which methods should be used to establish the diagnosis. The earliest retinal change is abnormal pigmentation around the macula, referred to as stippling or mottling, but there is a wide variation in normal macular appearance, and similar changes may occur in the aging eye $\mathrm{e}^{44}$ and have also been found in rheumatoid patients who have never received chloroquine. ${ }^{45}$ In the well-established chloroquine retinopathy there are concentric rings 
of hyperpigmentation and depigmentation around the macula producing a bull's eye appearance, but this configuration is not pathognomonic of chloroquine toxicity ${ }^{46}$ and was probably a chance finding in one of the patients reported in this study (Table 3 , case 5).

The unreliability of fundus examination may explain the multiplicity of other techniques which have been advocated in the diagnosis of chloroquine retinopathy. Visual field examination characteristically shows a pericentral scotoma to a red target, ${ }^{47}$ but there is a $6 \%$ incidence of red scotomata in the normal population, ${ }^{48}$ and accurate testing of visual acuity requires a co-operative patient. Tests of colour vision may be used as a screening test for macular damage, ${ }^{43}$ but some patients have normal colour vision in the presence of macular mottling and scotomata, ${ }^{68}$ and colour discrimination may be abnormal in rheumatoid patients who have never received chloroquine. ${ }^{49}$ There have been enthusiastic claims for the value of electroretinography ${ }^{50}$ and electro-oculography, ${ }^{51} 52$ but both tests may be normal even if macular pigmentation is grossly disturbed. ${ }^{313453}$ Serial recordings of the electrooculogram in the normal eye have shown variation with time, ${ }^{54}$ and in patients on chloroquine the individual variation is too great for the test to be of diagnostic value. ${ }^{55}$ Other tests that have been tried include fluorescein angiography, ${ }^{37}$ the macular dazzle or photostress test, ${ }^{956}$ and dark adaptometry, ${ }^{57}$ but abnormalities in these tesis may be age-related and are not specific for chloroquine retinotoxicity. 464958 The reliability of ophthalmic assessment might be improved by performing a battery of tests, ${ }^{6}$ but this would be time consuming and is impracticable for monitoring most patients. There is therefore no completely reliable means of diagnosing chloroquine retinopathy before serious visual impairment has occurred.

The review of published case reports on chloroquine retinopathy (Table 1) has confirmed that the major risk of visual impairment relates to those patients receiving a daily dose above $250 \mathrm{mg}$. There were only 13 reported cases of patients developing a permanent reduction in visual acuity on a daily dose of $250 \mathrm{mg}$, and 3 of these had previously received other antimalarials. Mayer ${ }^{26}$ reported the case of a 30-year-old woman with rheumatoid arthritis who developed impaired vision on $250 \mathrm{mg}$ daily but commented that she had received a total dose of $782 \mathrm{~g}$ over $3 \frac{1}{4}$ years, which means she was probably receiving $500 \mathrm{mg}$ daily. The case described by Reed and Campbell ${ }^{27}$ is not typical of chloroquine retinopathy because the patient developed a central scotoma and became blind but had a normal macula and fundus. The only patients who developed unquestionable chloroquine retinopathy with im- paired vision on a daily dose not exceeding $250 \mathrm{mg}$ and had not received other antimalarials are 4 cases described by Nylander, ${ }^{6} 2$ by Voipio and Karjalainen, ${ }^{8}$ and one each by Smith ${ }^{28}$ and Carlberg. ${ }^{40}$

The cases of chloroquine retinopathy reported to the CSM were unsuitable for retrospective analysis because the details of their ophthalmic examination were usually inadequate. The visual acuity was recorded in only 2 patients, one of whom developed a chloroquine retinopathy associated with impaired vision on a daily dose of $200 \mathrm{mg}$. Three other patients treated with chloroquine $250 \mathrm{mg}$ daily developed a retinopathy, but in 2 there was no comment on whether vision was impaired, though the third was stated to have developed partial blindness. There is therefore evidence from 2 cases reported to the CSM (Table 2, cases 1,2) that chloroquine may cause visual impairment even if the daily dose does not exceed $250 \mathrm{mg}$.

The results of the postal questionnaire completed by rheumatologists at 41 centres in Great Britain showed that serious retinal problems rarely occurred as a result of chloroquine therapy and visual impairment was reported in only 13 cases. Six patients had unequivocal evidence that their visual impairment was the result of a chloroquine retinopathy, but 5 of these had been receiving daily doses of chloroquine phosphate above $250 \mathrm{mg}$. In 3 other cases the visual impairment was probably coincidental to chloroquine therapy. There was therefore only 1 patient (Table 3 , case 7) on chloroquine 250 $\mathrm{mg}$ daily who developed impaired vision as a result of treatment.

The postal questionnaire was completed by ophthalmologists at 33 centres. Eight patients treated with chloroquine developed impaired vision, but details were available in only 3 , and only 1 of these (Table 4 , case 2 ) had been receiving chloroquine $250 \mathrm{mg}$ daily. There was therefore little evidence from ophthalmic sources that chloroquine use in Great Britain is causing visual problems.

The purpose of this study was to establish whether there is a safe daily dose of chloroquine which does not lead to impaired vision. From a review of cases from 3 different sources, namely, published case reports, reports to the CSM, and from personal communication with British rheumatologists and ophthalmologists, the risk of visual impairment associated with chloroquine therapy appears to be very small provided the daily dose does not exceed $250 \mathrm{mg}$. But occasional cases may occur at this dose level, so that ophthalmic supervision should still be undertaken.

I thank the members of the Heberden Society and Othalmological Society of the United Kingdom for their replies to the questionnaire on chloroquine retinopathy. 


\section{References}

1 Freedman A, Steinburg V L. Chloroquine in rheumatoid arthritis: a double blindfold trial of treatment for one year. Ann Rheum Dis 1960; 19: 243-50.

2 Popert A J, Meijers K A E, Sharp J, Bier F. Chloroquine diphosphate in rheumatoid arthritis: a controlled trial. Ann Rheum Dis 1961; 20: 18-33.

3 Rothermich N O. Coming catastrophes with chloroquine? Ann Intern Med 1964; 61 : 1203-5.

4 Scherbel A L, Mackenzie A H, Nousek J E, Atdjian M. Ocular lesions in rheumatoid arthritis and related disorders with particular reference to retinopathy. $N$ Engl J Med 1965; 273: 360-6.

5 Fuld H. Retinopathy following chloroquine therapy. Lancet 1959; ii: 617-8.

6 Nylander U. Ocular damage in chloroquine therapy. Acta Ophthalmol (Kbh) 1967; suppl 92:1-71.

7 Arden G B, Kolb H. Antimalarial therapy and early retinal changes in patients with rheumatoid arthritis. $\mathrm{Br}$ Med J 1966; i: 270-3.

8 Voipio H, Karjalainen K. Retinal and visual field changes in chloroquine retinopathy. Acta Ophthalmol (Kbh) 1967; 45: $150-8$.

9 Carr R E, Henkind P, Rothfield N, Siegel I M. Ocular toxicity of antimalarial drugs. Am J Ophthalmol 1968; 66: 738-44.

10 Rubin $M$, Bernstein $H$, Zvaifler N. Studies on the pharmacology of chloroquine with recommendation for the treatment of chloroquine retinopathy. Arch Ophthalmol 1963; 70: 474-81.

11 Bernstein H, Ginsberg J. The ocular pathology of chloroquine retinopathy. Arch Ophthalmol 1964; 71: 238-45.

12 Noell W K, Walker V S, Kang B S. Retinal damage by light in rats. Invest Ophthalmol Visual Sci 1966; 5: 450-73.

13 Rubin M, Slonicki A. A mechanism for the toxicity of chloroquine. Arthritis Rheum 1966;9: 537.

14 Mackenzie A H, Scherbel A L. A decade of chloroquine maintenance therapy: rate of administration governs incidence of retinopathy. Arthritis Rheum 1968; 11: 496.

15 Marks J S, Power B J. Is chloroquine obsolete in the treatment of rheumatic disease? Lancet 1979 ; i: 371-3.

16 Cambiaggi A. Unusual ocular lesions in a case of systemic lupus erythematosus. Arch Ophthalmol 1957; 57: 451-3.

17 Hobbs H E, Sorsby A, Freedman A. Retinopathy following chloroquine therapy. Lancet 1959 ; ii: 478-80.

18 Wells G C. Amblyopia in lupus erythematosus. Proc $R$ Soc Med 1959; 52: 1031-2.

19 Hobbs H E, Eadie S P, Somerville F. Ocular lesions after treatment with chloroquine. Br J Ophthalmol 1961; 45: 284-97.

20 Richards R D, Wilson W R. Retinopathy associated with chloroquine phosphate therapy. Am J Med 1961; 31: 141-3.

21 Ellsworth $\mathrm{R}$ J, Zeller $\mathrm{R}$ W. Chloroquine induced retinal damage Arch Ophthalmol 1961; 66: 269-72.

22 Price L. Retinopathy associated with chloroquine. Trans Ophthalmol Soc Aust 1961; 21 : 35-8.

23 George J B, Mitchell P C. Chloroquine retinopathy. $J \boldsymbol{R}$ Army Med Corps 1962; 108: 87-90.

24 Ormrod $\mathrm{J} N$. Two cases of chloroquine induced retinal damage. Br Med J 1962; i: 918-21.

25 Penner $\mathbf{R}$, Somers $\mathrm{K}$. Bilateral macular degeneration associated with chloroquine therapy. Am J Ophthalmol $1962 ; 54: 381-5$.
26 Mayer W. Chloroquine retinopathy. Am J Ophthalmol 1962; 53: 769-74.

27 Reed H, Campbell A A. Central scotoma following chloroquine therapy. Can Med Assoc J 1962; 86: 176e8.

${ }^{28}$ Smith J L. Chloroquine macular degeneration. Arch Ophthalmol 1962; 68: 186-90.

29 Jansson F. Electroretinographic changes in chloroquine therapy. Acta Ophthalmol (Kbh) 1962; suppl 70: 252-7.

30 Algvere P, Carberg O, Ericson L. Retinal damage in chloroquine therapy. Acta Ophthalmol (Kbh) 1963; 41: 469-72.

31 Okun E, Gouras P, Berstein H, Von Sallman L. Chloroquine retinopathy. A report of eight cases with ERG and dark adaptation findings. Arch Ophthalmol 1963; 69: 59-71.

32 Elliott J H, Mills J B. Chloroquine retinopathy. J Okla State Med Assoc 1963; 56: 391-6.

${ }^{33}$ Crews $\mathbf{S}$ J. Chloroquine retinopathy with recovery in the early stages. Lancet 1964 ; ii: 436-8.

${ }^{34}$ Henkind P, Carr R E, Siegel I M. Early chloroquine retinopathy. Clinical and functional findings. Arch Ophthalmol 1964; 71 : 157-65.

35 Lewis P M. Chloroquine blindness. Am J Ophthalmol $1964 ; 57: 677-8$.

36 Lloyd L A, Hiltz J W. Ocular complications of chloroquine therapy. Can Med Assoc J 1965; 92: 508-13.

37 Kearns T P, Hollenhorst R W. Chloroquine retinopathy. Arch Ophthalmol 1966; 76: 378-84.

38 Burns R P. Delayed onset chloroquine retinopathy. N Engl J Med 1966; 275: 693-6.

39 Crews S J. The prevention of drug induced retinopathies. Trans Ophthalmol Soc UK 1966; 86: 63-76.

40 Carlberg $O$. Three cases of chloroquine retinopathy. A follow up investigation. Acta Ophthalmol (Kbh) 1966; 44: 367-74.

41 Brinkley J R, Dubois E L, Ryan S J. Long term course of chloroquine retinopathy after cessation of medication. Am J Ophthalmol 1979; 88: 1-11.

42 Henkind $\mathbf{P}$, Rothfield N. Ocular abnormalities in patients treated with synthetic antimalarial drugs. $N$ Engl $J$ Med 1963; 269: 433-9.

43 Nozik R A, Weinstock F, Vignos P. Ocular complications of chloroquine. Am J Ophthalmol 1964; 58: 774-8.

44 Kornzweig A L, Feldstein M. Studies of the eye in old age. Am J Ophthalmol 1950; 33: 243-7.

45 Bals B. Chloroquine retinopathy and its incidence in patients with rheumatoid arthritis. Acta Rheumatol Scand 1964; 10: 227-40.

46 Weise E E, Yannuzzi L A. Ring maculopathies mimicking chloroquine retinopathy. Am J Ophthalmol 1974; 78: 204-10.

47 Percival S P B, Meanock I. Chloroquine: ophthalmological safety and clinical assessment in rheumatoid arthritis. Br Med J 1968; iii: 579-84.

48 Percival S P B, Behrman J. Ophthalmological safety of chloroquine. Br J Ophthalmol 1969; 53: 101-9.

49 Fiechtner J J, Berry B J, Simkin P A. Vision and taste deficits in rheumatoid arthritis. Arthritis Rheum 1980; 23: 672 .

50 Schmidt B, Muller L W. Electroretinographic examinations following the application of chloroquine. Acta Ophthalmol (Kbh) 1962; suppl 70: 245-51.

51 Arden G B, Friedmann A, Kolb H. Anticipation of chloroquine retinopathy. Lancet 1962; i: 1164-5.

52 Copeman $M P$ W, Cowell $T \mathbf{T}$, Dallas $\mathbf{N}$ L. Screening test for chloroquine retinopathy. Lancet 1964; i: $1369-70$. 
53 Adlakha D, Crews S J, Shearer A C I, Tonks E L. Electro diagnosis in drug induced disorders of the eye. Trans Ophthalmol Soc UK 1967; 87: 267-84.

54 Kelsey $\mathbf{J} \mathbf{H}$. Variations in the normal electro-oculogram. Br J Ophthalmol 1967; 51 : 44-9.

55 Gouras P, Gunkel R. The EOG in chloroquine and other retinopathies. Arch Ophthalmol 1963; 70: 629-39.
56 Henkind P, Siegel I M. The 'scotometer'-a device for measuring macular recovery time. Am J Ophthalmol $1967 ; 64: 314-5$.

57 Carr R, Gouras F, Gunkel R. Chloroquine retinopathy. Arch Ophthalmol 1966; 75: 171-8.

${ }^{58}$ Severin S L, Tour R L, Kershaw R H. Macular function and the photostress test. Arch Ophthalmol 1966;77: 163-7. 